\title{
Patterns of Human Diversity, within and among Continents, Inferred from Biallelic DNA Polymorphisms
}

\author{
Chiara Romualdi, ${ }^{1,2,7}$ David Balding, ${ }^{3,8}$ Ivane S. Nasidze, ${ }^{4}$ Gregory Risch, ${ }^{5}$ \\ Myles Robichaux, ${ }^{5}$ Stephen T. Sherry, ${ }^{5}$ Mark Stoneking, ${ }^{4}$ Mark A. Batzer, ${ }^{5,6}$ \\ and Guido Barbujani ${ }^{1,9}$
}

${ }^{1}$ Department of Biology, University of Ferrara, Ferrara I-44100, Italy; ${ }^{2}$ Department of Statistics, University of Padua, Padua I-35121, Italy; ${ }^{3}$ Department of Applied Statistics, University of Reading, Reading RG6 6FN, United Kingdom; ${ }^{4}$ Max Planck Institute for Evolutionary Anthropology, Leipzig, D-04103 Germany; ${ }^{5}$ Department of Pathology, Stanley S. Scott Cancer Center, Louisiana State University Health Sciences Center, New Orleans, Louisiana 70112, USA; ${ }^{6}$ Department of Biological Sciences, Biological Computation and Visualization Center, Louisiana State University, Baton Rouge, Louisiana 70803, USA

Previous studies have reported that about $85 \%$ of human diversity at Short Tandem Repeat (STR) and Restriction Fragment Length Polymorphism (RFLP) autosomal loci is due to differences between individuals of the same population, whereas differences among continental groups account for only $10 \%$ of the overall genetic variance. These findings conflict with popular notions of distinct and relatively homogeneous human races, and may also call into question the apparent usefulness of ethnic classification in, for example, medical diagnostics. Here, we present new data on 21 Alu insertions in 32 populations. We analyze these data along with three other large, globally dispersed data sets consisting of apparently neutral biallelic nuclear markers, as well as with a $\beta$-globin data set possibly subject to selection. We confirm the previous results for the autosomal data, and find a higher diversity among continents for Y-chromosome loci. We also extend the analyses to address two questions: (1) whether differences between continental groups, although small, are nevertheless large enough to confidently assign individuals to their continent on the basis of their genotypes; (2) whether the observed genotypes naturally cluster into continental or population groups when the sample source location is ignored. Using a range of statistical methods, we show that classification errors are at best around $30 \%$ for autosomal biallelic polymorphisms and $27 \%$ for the $Y$ chromosome. Two data sets suggest the existence of three and four major groups of genotypes worldwide, respectively, and the two groupings are inconsistent. These results suggest that, at random biallelic loci, there is little evidence, if any, of a clear subdivision of humans into biologically defined groups.

In various areas of applied genetics, it is customary to regard the human species as divided in distinct and objectively recognizable groups. Forensic scientists compare DNA profiles from the place of a crime with databases from the general population, usually grouped into broad racial categories (for instance, African-American, European-American, Asian, and Hispanic), to estimate the probability that an unrelated individual would have the identical DNA profile. The markers chosen for DNA profiling are considered to be essentially uniform across populations of the same category. Although the existence of problems with group definition has been acknowledged (e.g., Weir 2001), the fact that some individuals may not be easy to allocate to any such group is usually regarded as unimportant (National Research Council 1992; Lander and Budowle 1994; Morton 1994; Roeder 1994; Gill and Evett 1995). In clinical practice, a correlation of racial

7Present addresses: CRIBI, Biotechnology Centre, University of Padua, Padua I-35121 Italy; ${ }^{8}$ Department of Epidemiology and Public Health, Imperial College, St. Mary's campus, Norfolk Place, London W2 1PG, United Kingdom.

${ }^{9}$ Corresponding author. University of Ferrara, Department of $\mathrm{Bi}$ ology, via L. Borsari 46, I-44100 Ferrara, Italy

E-MAIL bjg@unife.it; FAX (+39) 0532249761.

Article and publication are at http://www.genome.org/cgi/doi/10.1101/ gr.214902. affiliation, as assessed from skin color, facial characteristics, hair texture, and so forth, with disease pathology and drug response is widely believed to exist. A PubMed search with the keywords "human races" (January 10, 2002) yielded 34,143 papers, including Benar et al. (2001), Estrada and Billett (2001), Hartz et al. (2001), Hoffman et al. (2001), and Shaw and Krause (2001).

In contrast, population studies have suggested that genetic variation is essentially continuous through space among humans, and have failed to identify a set of genetically distinct and internally homogeneous groups. Regardless of whether estimated at the protein (Lewontin 1972; Latter 1980), craniometric (Relethford 1994), or DNA (Barbujani et al. 1997, Jorde et al. 2000) level, individual differences between members of the same population have been reported to account for about $85 \%$ of the overall genetic diversity, and differences between populations within the same continent account for a further $5 \%$ to $10 \%$. Only about $10 \%$ of variation can be assigned to differences between continental groups.

The existence of such different views in related areas of science has probably more than one cause, but a clearer picture of human genetic diversity is necessary to at least reduce the levels of disagreement. One open problem has to do with the exact amount of genetic diversity that can be attributed to 
the various levels of population subdivision. The figures mentioned earlier were estimated from populations that were well separated in space, and therefore they may exaggerate the between-group component of genetic variance. On the other hand, most of the markers studied are thought to be approximately neutral, which may have the opposite effect if between-group variation reflects adaptation to spatially variable factors such as climate. A second question is whether the apparently continuous distribution of genetic variation implies in practice that meaningful groups cannot be identified, as has been argued by Templeton (1999). In fact, genetic variances among groups, although small, are significantly greater than zero at several loci. That may mean that, by jointly considering many loci, distinct groups may emerge, even though those groups cannot be discriminated at the single-locus level.

We shall start by borrowing a definition from evolutionary and conservation genetics. In those fields, races or subspecies are defined as recognizable lineages within a species that have diverged genetically because mating barriers have separated them for a sufficiently long time (Templeton 1999; see also Pennock and Dimmick 1997). We shall ask if there is genetic evidence that the human species is subdivided in groups of that kind. To address that question, we consider fast-evolving DNA markers as less than optimal. Indeed, STR and mitochondrial polymorphisms, such as most of those considered by Barbujani et al. (1997) and Jorde et al. (2000), have high mutation rates, and hence their patterns of variation are likely to reflect relatively recent divergence. Evidence of long-term subdivision among populations, if any, is more likely to be found by analyzing slow-mutating DNA sites, typically biallelic polymorphisms, which presumably evolved only once ("unique-event polymorphisms"; see Markovtsova et al. 2000) in human history.

For that purpose, we typed 21 Alu insertion polymorphisms in population samples from five continents, and we analyzed published biallelic DNA polymorphism data at several other nuclear loci, both autosomal and Y linked. On the five data sets thus assembled, we estimated the components of variance that can be attributed to differences between individuals, between populations of the same continent, and between continental groups, and we compared our estimates with previously published values. In addition, we investigated with what degree of accuracy individuals can be attributed to their continent on the basis of their genotypes, and which are the most likely clusters of individuals that can be inferred from multilocus genotypes, regardless of their geographical provenance.

\section{RESULTS AND DISCUSSION}

\section{Alu Insertion Frequencies}

Table 1 reports the frequencies of the alternative alleles (presence or absence of the insertion, the latter representing the likely ancestral state; Watkins et al. 2001) at the 21 loci typed in this study. The standardized genetic variance, $\mathrm{F}_{\mathrm{st}}$, summarizes for each locus the global differentiation among populations of all continents. Values of $\mathrm{F}_{\text {st }}$ close to $15 \%$ are often observed in worldwide analyses of humans (Cavalli-Sforza et al. 1994). Hence, most Alu loci of this study display what can be considered "normal" levels of interpopulation diversity.

\section{Genetic Differences among Continental Groups}

For both Alu-insertion data sets (Alu8 and Alu21, comprising information, respectively, on 8 and 21 loci), an analysis of molecular variance, AMOVA (Excoffier et al. 1992) was run once for each locus, and once for the multilocus genotypes. The Y-chromosome (Y98, Y99 data sets) and $\beta$-globin (BGL data set) data were each subjected to independent runs of AMOVA using all the available sequence information. For most Alu loci, for the compound Alu genotypes, and for the BGL data set, around $80 \%$ of the overall genetic diversity is allocated to differences among members of the same sample (Table 2). About $10 \%$ is attributed to differences among populations within the same continent (less for the BGL data set, where most continents, however, were represented by only one population), and the rest, a little over 10\%, to differences among continents. The exceptions are one Alu locus (FXIIIB) and the Y chromosome, which show a lower component of variance within populations (between 42 and 46\%) and a higher component between continental groups (close to $40 \%$ ). Even for these loci, the greatest fraction of genetic variance occurs within populations.

In two previous Y-chromosome studies based, respectively, on a combination of SNP and STR markers, Hammer et al. (2001) and Jorde et al. (2000) estimated lower variances among continents and higher variances within populations. In Jorde et al.'s (2000) STR study, in particular, variances between continents were practically zero. The simplest explanation is that different mutational mechanisms generate diversity at biallelic sites and at STR loci. Because of the higher mutation rate of the latter (average $2.8 \times 10^{-3}$ per locus per generation; Kayser and Sajantila 2001) and of probable constraints to allele size (Deka et al. 1999), it seems that most populations tend to approach a common allelic distribution for those markers. Conversely, biallelic polymorphisms mutate more slowly (about $5 \times 10^{-7}$ per site per generation; Jobling et al. 1997), and therefore their distribution reflects more the effects of demographic history than those of mutation.

The genetic variances among continents inferred in this study from the Y-chromosome data sets are greater than those observed for autosomal markers. The same is true, to a lesser extent, of mtDNA, where the fraction of variance between continents, $12.5 \%$, is still higher than for the nuclear genes of the same study (Seielstad et al. 1998). These results were expected under a model of neutral evolution, driven by genetic drift and gene flow. If selection is negligible, the genetic variance among populations $\left(\mathrm{F}_{\mathrm{st}}\right)$ tends to reach an equilibrium value, which, in Wright's (1969) classical model, is inversely proportional to $N$, the population size, times $m$, the gene flow rate. We do not know whether our populations are at equilibrium, but $N$ of mitochondrial and Y-chromosome loci is one-fourth that of autosomal loci, so that the impact of drift is greater on the former. Therefore, it seems that the genetic drift that has been going on since the (apparently recent and incomplete) separation of continental human groups has so far been able to generate appreciable differences only at uniparentally transmitted loci.

\section{Inferring the Geographic Origin of a Genotype}

To test the extent to which continents are associated with specific sets of alleles, we initially disregarded the geographic information. We took one genotype at a time, and attributed it to its most likely continent according to eight methods of discriminant analysis, three of them parametric and five nonparametric (listed in the caption to Table 3). Then we calculated the rate of misassignment for each method, that is, the 


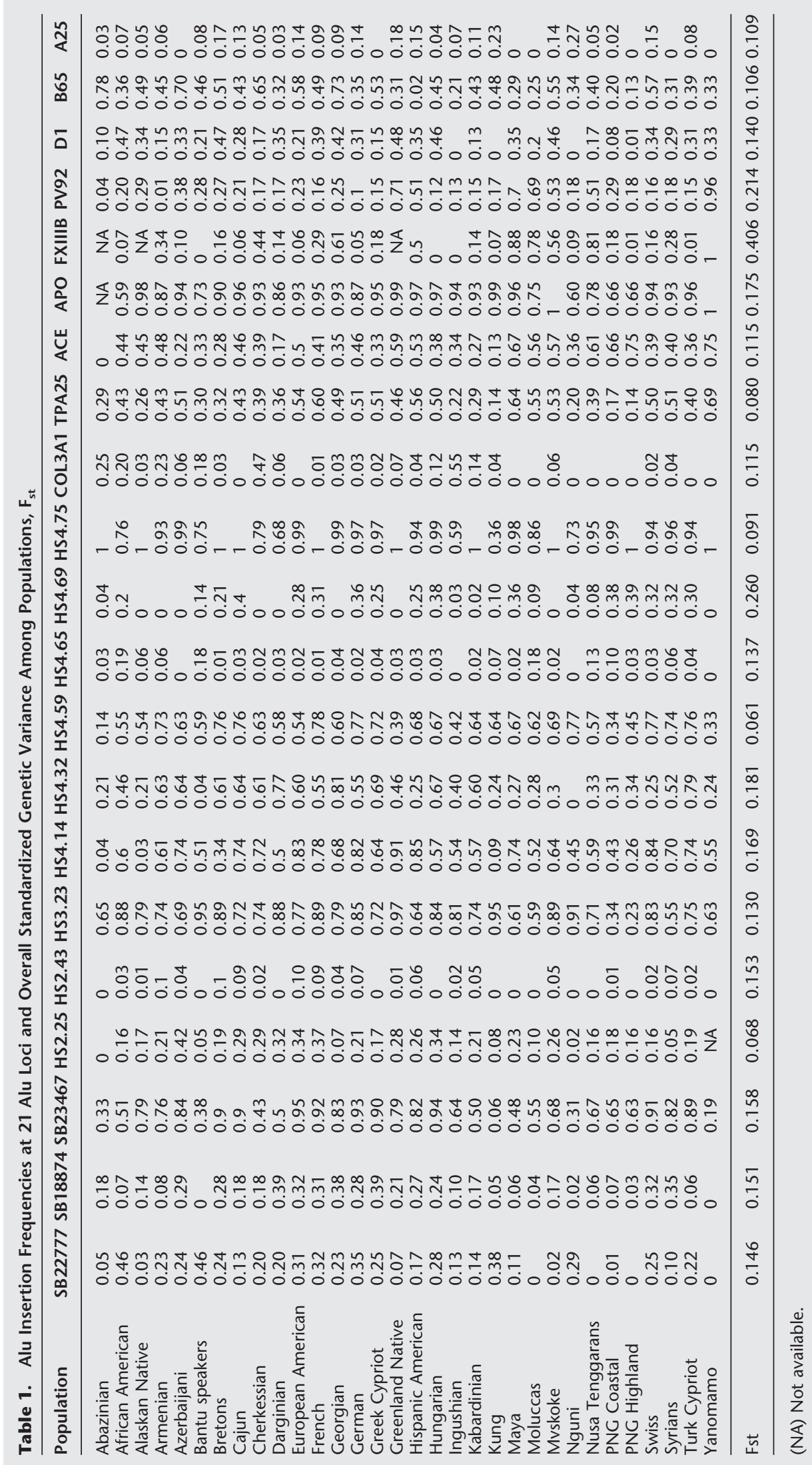

604 Genome Research 
Table 2. Components of Genetic Variance (\%) at Three Levels of Population Subdivision

\begin{tabular}{lccc}
\hline Locus & $\begin{array}{c}\text { Between } \\
\text { continents }\end{array}$ & $\begin{array}{c}\text { Between } \\
\text { populations } \\
\text { within continents }\end{array}$ & $\begin{array}{c}\text { Within } \\
\text { populations }\end{array}$ \\
\hline TPA & 5.97 & 4.84 & 89.19 \\
ACE & 12.05 & 7.12 & 80.83 \\
APO & 7.26 & 13.77 & 78.97 \\
FXIIB & 38.17 & 14.97 & 46.86 \\
PV92 & 18.51 & 16.22 & 65.27 \\
D1 & 4.80 & 8.89 & 86.32 \\
B65 & 1.53 & 8.24 & 90.23 \\
A25 & 2.27 & 9.26 & 88.47 \\
SB22777 & 13.27 & 6.70 & 80.03 \\
SB18874 & 8.39 & 8.39 & 83.22 \\
SB323467 & 15.54 & 19.32 & 65.14 \\
HS2.25 & 6.29 & 7.29 & 86.42 \\
HS2.43 & 1.31 & 5.44 & 93.25 \\
HS3.23 & 19.05 & 4.27 & 76.68 \\
HS4.14 & 6.22 & 17.18 & 76.60 \\
HS4.32 & 11.90 & 12.47 & 75.63 \\
HS4.59 & 3.90 & 5.71 & 90.39 \\
HS4.65 & 4.10 & 5.71 & 90.19 \\
HS4.69 & 8.81 & 22.80 & 68.39 \\
HS4.75 & 20.76 & 16.91 & 62.33 \\
COL3A1 & -4.68 & 39.36 & 65.32 \\
\hline Alu8 overall & 12.70 & 9.96 & 77.34 \\
Alu21 overall & 8.90 & 8.22 & 82.87 \\
\hline B-globin & 17.80 & 2.80 & 79.39 \\
\hline Y98 & 38.99 & 14.71 & 46.30 \\
Y99 & 40.11 & 17.39 & 42.50 \\
\hline
\end{tabular}

The Alu8 data set is used for the first eight loci; the Alu21 data set is used for the remainder.

percentage of individuals who were wrongly allocated (Table $3)$. Among nuclear loci, the worst results are obtained for the BGL data set: more than half of the genotypes are misclassified. The Alu data sets give better results, as would be anticipated for multilocus data. The NNET and LOG methods each give error rates of $38 \%$ and $32 \%$ for Alu 8 and Alu21, whereas

Table 3. Percentage of Misclassification in Discriminant Analysis by Data Set and Method Used

\begin{tabular}{|c|c|c|c|c|c|c|c|c|}
\hline \multirow[b]{2}{*}{ Data set } & \multicolumn{3}{|c|}{ Parametric } & \multirow[b]{2}{*}{ NNET } & \multicolumn{3}{|c|}{ Nonparametric } & \multirow[b]{2}{*}{ RM } \\
\hline & LDA & LOG & QDA $^{\mathrm{a}}$ & & KER & $1 \mathrm{NN}$ & $3 N N$ & \\
\hline u21 & 40 & 32 & NA & 38 & 37 & 32 & 36 & 30 \\
\hline Alu8 & 49 & 35 & 51 & 35 & 49 & 42 & 38 & 37 \\
\hline$\beta$-globin & 58 & 63 & 65 & 52 & 58 & 52 & 53 & 50 \\
\hline Y98 & 79 & 71 & 70 & 27 & 61 & 27 & 27 & 40 \\
\hline Y99 & 83 & 81 & 73 & 27 & 43 & 27 & 27 & 37 \\
\hline
\end{tabular}

aThe QDA method could not be used for the Alu21 data set because of the insufficient number of individuals with the complete, 21-locus genotype in some samples.(LDA) Linear discriminant analysis; (LOG) logistic discriminant analysis; (QDA) quadratic discriminant analysis; (NNET) neural networks; (KER) nonparametric discriminant analysis with Gaussian kernel; ( $1 \mathrm{NN}$ and $3 \mathrm{NN}$ ) nonparametric discriminant analysis with, respectively, 1 and 3 nearest neighbors; (RM) Rannala and Mountain (1997) method. with the RM method, $37 \%$ and $30 \%$ of the genotypes, respectively, were misclassified.

For the Y-chromosome data sets, the parametric methods again perform poorly, with misclassification rates at least $70 \%$. However, NNET, $1 \mathrm{NN}$, and $3 \mathrm{NN}$ each give an error rate of $27 \%$, better than the $40 \%$ obtained using the RM method and also better than the error rates obtained using the same methods for the multilocus data sets. Although only a single locus, the Y chromosome is relatively powerful for discrimination because its between-group variance is higher, as revealed by AMOVA (this study; Hammer et al. 2001) and by previous independent studies (Underhill et al. 2000).

Table 4 is an example of the so-called confusion matrix for the classification of individuals from the Alu8 data set using the method that gave the lowest rate of misclassification, NNET. The entry in row $i$ and column $j$ gives the number of individuals drawn from continent $i$ that are classified into continent $j$ (so that the diagonal entries correspond to correct classifications). Table 5 gives the same information for the Y99 data set, again using the method giving the most accurate results for that data set, $1 \mathrm{NN}$. At the bottom of Table 5, Asia was further subdivided in two subregions, which caused an increase in the misclassification rate. Overall, the results indicate poor discrimination, with even the best method and data set leading to nearly $30 \%$ misclassification. Even our relatively large data sets do not suffice to allow accurate assignment of individuals into their continent.

To test how the number of loci considered affects these results, we repeatedly (500 times) analyzed subsets of the Alu21 data set, consisting of increasing numbers of loci chosen at random from the 21 available. The rate of misclassification decreased rapidly at the very beginning, but then leveled off (Fig. 1), suggesting that the error rate will not become zero, even further increasing the number of loci. This supports the view that error rate reflects, in part, factors other than the limited number of loci considered, including genetic exchanges leading to extensive allele sharing among populations. In principle, these results might be explained by the presence of a few hybrid populations at the boundaries between continents, but that proves not to be the case. If one looks at the geographic origin of the misclassified individuals (Table 6 is an example), it is evident that many other populations contain genotypes or haplotypes that discriminant analysis classifies along with those of another continent. For

Table 4. Confusion Matrix from the NNET Discriminant Analysis of the Alu8 Data Set

\begin{tabular}{lrrrcccr}
\hline & \multicolumn{6}{c}{ Assigned to } \\
\cline { 2 - 7 } Sample & Africa & Europe & Asia & Americas & Australia & Total \\
\hline Africa & $28 \%$ & $52 \%$ & $13 \%$ & $0 \%$ & $7 \%$ & 138 \\
Europe & $4 \%$ & $72 \%$ & $22 \%$ & $0.4 \%$ & $1.6 \%$ & 457 \\
Asia & $2 \%$ & $18 \%$ & $73 \%$ & $4 \%$ & $3 \%$ & 580 \\
Americas & $0 \%$ & $6 \%$ & $75 \%$ & $19 \%$ & $0 \%$ & 89 \\
Australia & $13 \%$ & $15 \%$ & $21 \%$ & $0 \%$ & $51 \%$ & 67 \\
\hline Total & 81 & 517 & 627 & 42 & 64 & 1331 \\
\hline
\end{tabular}

Each cell contains the fraction of individuals whose origin is in the row continent, who were allocated to the continent indicated by the column label; correct allocations are therefore on the main diagonal. Totals are numbers of individuals. 
Romualdi et al.

Table 5. Confusion Matrices from Two 1 NN Discriminant Analyses of the Y99 Data Set

\begin{tabular}{|c|c|c|c|c|c|c|c|}
\hline \multirow[b]{2}{*}{ Sampled in } & \multicolumn{7}{|c|}{ Assigned to } \\
\hline & Africa & Europe & & & Americas & Australia & Total \\
\hline Africa & $90 \%$ & $5 \%$ & & $\%$ & $0 \%$ & $0 \%$ & 348 \\
\hline Europe & $42 \%$ & $5 \%$ & & & $0 \%$ & $0 \%$ & 175 \\
\hline Asia & $3 \%$ & $1 \%$ & & & $0 \%$ & $0 \%$ & 1168 \\
\hline Americas & $5 \%$ & $1 \%$ & & & $53 \%$ & $0 \%$ & 374 \\
\hline Australia & $0 \%$ & $0 \%$ & & & $0 \%$ & $0 \%$ & 133 \\
\hline \multirow[t]{2}{*}{ Total } & 440 & 47 & & & 205 & 0 & 2198 \\
\hline & \multicolumn{7}{|c|}{ Assigned to } \\
\hline Sampled in & Africa & Europe & $\begin{array}{c}\text { NC } \\
\text { Asia }\end{array}$ & $\begin{array}{c}\text { SE } \\
\text { Asia }\end{array}$ & Australia & Americas & Total \\
\hline Africa & $90 \%$ & $5 \%$ & $3 \%$ & $2 \%$ & $0 \%$ & $0 \%$ & 348 \\
\hline Europe & $42 \%$ & $5 \%$ & $51 \%$ & $2 \%$ & $0 \%$ & $0 \%$ & 175 \\
\hline NC Asia & $2 \%$ & $1 \%$ & $75 \%$ & $22 \%$ & $0 \%$ & $0 \%$ & 801 \\
\hline SE Asia & $6 \%$ & $1 \%$ & $20 \%$ & $73 \%$ & $0 \%$ & $0 \%$ & 367 \\
\hline Americas & $5 \%$ & $1 \%$ & $41 \%$ & $0 \%$ & $0 \%$ & $53 \%$ & 374 \\
\hline Australia & $0 \%$ & $0 \%$ & $31 \%$ & $69 \%$ & $0 \%$ & $0 \%$ & 133 \\
\hline Total & 440 & 47 & 960 & 546 & 0 & 205 & 2198 \\
\hline
\end{tabular}

from Northern and Central America (Tanana, Cheyenne, Pima, Havasupay, and Pueblos), whereas the misclassification rate decreases as one moves southward. The results are less clear for the Alu data sets (data not given), where we could not recognize a clear pattern of misclassification. This could be due to factors such as the lower number of populations available, their spatial distribution, or the already discussed effect of population sizes. However, a crucial factor to consider is the absence of recombination for Y-chromosome markers, so that migrant $\mathrm{Y}$ chromosomes may convey evidence of their source population for many generations.

Indeed, the greater differences among continents observed for $\mathrm{Y}$ chromosome markers (all of them mapping on the nonrecombining portion of the chromosome) did not lead to a much better allocation of genotypes of unknown origin. Haplotypes are transmitted as a

the Y99 data set, there seems to be a tendency to misclassify individuals from populations at nearby latitudes. Seventyfour European haplotypes are wrongly allocated to Africa, and they are mostly Greeks and Italians. Conversely, among the 90 Europeans that are allocated to North-Central Asia, most are British, Russians, and Germans. In the Americas, most of the 152 individuals allocated to North-Central Asia come

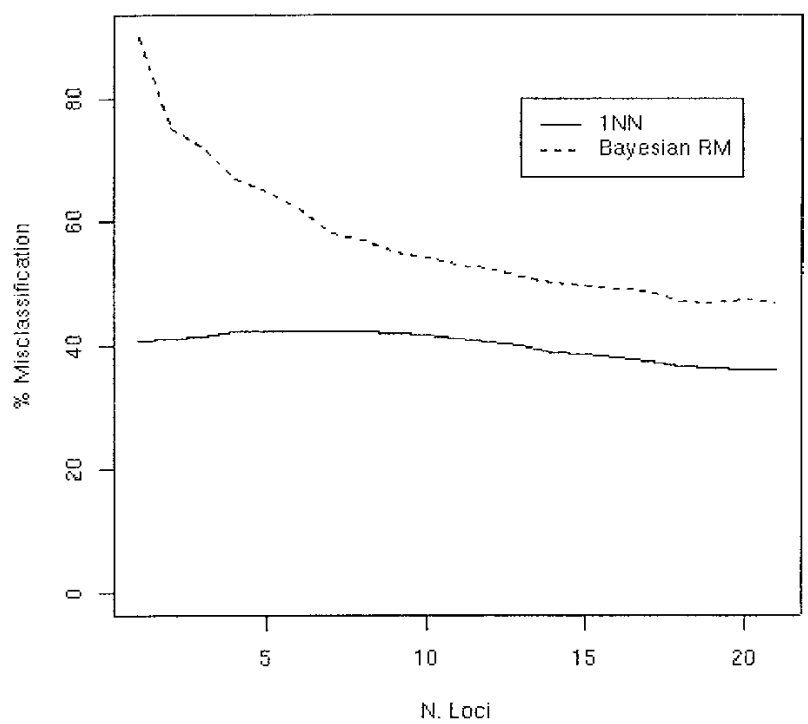

Figure 1 Relationships between the misclassification rate of the discriminant analysis ( $Y$ axis) and the number of loci used for the discrimination ( $X$ axis), using two different methods and the Alu21 data set. The graphs represent averages over 500 independent replicates. (Broken line) 1NN, (solid line) RM with Bayesian approach. The loci were added sequentially by Montecarlo randomization, so that their order is not expected to affect the misclassification rate. single unit, and can in principle be followed through time and place. On the other hand, though, each of those haplotypes can be regarded as one variant of a multiallelic locus, and the power of discriminant analysis increases with the number of independent loci considered (compare the Alu and Y-chromosome data sets in Table 3).

The low accuracy of discriminant analysis does not depend on a poor definition of the continental groups considered. In fact, the more groups are considered, the higher the misclassification rate. Compare, for example, the top and the bottom of Table 5; one-fifth of North-Central Asians were classified as South-Eastern Asians and vice versa, when these two groups were separately considered. At a subcontinental level, inferring the geographic origin of a person from her/his genotype becomes more complicated, and therefore it seems unlikely that classification errors would be reduced by choosing among a higher number of potential origins.

Most misclassified individuals were assigned to Europe and Asia in Table 4 (Alu8 data set), and to Africa and Asia in Table 5 (Y99 data set). Many individuals from Australia and the Americas were attributed to Asia, where the first settlers came from, and 13\% (Table 4) of Australians, intriguingly, to Africa. These observations suggest that the distribution of misclassified individuals reflects, at least in part, past population movements. We are currently developing a formal model to infer past migrations from the results of discriminant analysis.

\section{Inferring Population Structure from Genotypes}

So far we have been trying to assign individuals to groups defined a priori on the basis of geography. An alternative is to identify groups a posteriori on the basis of genotypes, namely, to cluster genotypes until a certain number of genetically homogeneous groups is defined. The program STRUCTURE (Pritchard et al. 2000) infers the most likely number of such groups, and assigns individuals to each of them, on the basis 
Table 6. Origin of Misclassified Individuals, Y99 Data Set

\begin{tabular}{|c|c|c|}
\hline Assigned to & Sampled in ${ }^{a}$ & Detailed origin of the misclassified individuals ${ }^{a}$ \\
\hline Africa & $42 \%$ Europe & 19\% British, 38\% Germans, 49\% Italians, 76\% Greek, 17\% Russians \\
\hline NC Asia & $51 \%$ Europe & $78 \%$ British, 56\% Germans, $41 \%$ Italians, $21 \%$ Greek, $73 \%$ Russians \\
\hline NC Asia & $20 \%$ SE Asia & $7 \%$ Japanese, $9 \%$ Taiwanese, $12 \%$ S. Chinese, $25 \%$ Korean, $36 \%$ Indonesians, 3\% S. Asian, $52 \%$ Indians \\
\hline NC Asia & $31 \%$ Australia & $58 \%$ Australians, $11 \%$ PNG, 33\% Melanesians \\
\hline NC Asia & $41 \%$ Americas & $\begin{array}{c}25 \% \text { A. Eskimos, } 45 \% \text { I. Eskimos, } 50 \% \text { Tanana, } 45 \% \text { Navajos, } 77 \% \text { Cheyenne, } 50 \% \text { Havasupay, } 58 \% \text { Pima, } \\
50 \% \text { Pueblos, } 20 \% \text { Zapotecs, } 18 \% \text { Ngobe, } 20 \% \text { Wounan, } 10 \% \text { Mixtecs, } 50 \% \text { Wayus, } 33 \% \text { Chileans }\end{array}$ \\
\hline SE Asia & $22 \%$ NC Asia & $\begin{array}{l}37 \% \text { Komi, } 55 \% \text { F. Nentsi, } 73 \% \text { T. Nentsi, 6\% Buryats, 2\% S. Eskimos, } 41 \% \text { M. Evenks, } 4 \% \text { Oroquens, 5\% } \\
\text { Yakuts, } 8 \% \text { Koriats, 34\% Mongolians, 10\% Altais, 93\% Tibetans, 23\% Kazaks, 7\% Selkups }\end{array}$ \\
\hline SE Asia & $69 \%$ Australia & $42 \%$ Australians, $89 \%$ PNG, $67 \%$ Melanesians \\
\hline
\end{tabular}

aFigures are percentages of individuals misclassified over the continent's or the population's totals, respectively.

of probabilities estimated from a set of independently transmitted loci. Because Y-chromosome markers are genetically linked, this approach was suitable to analyze only the Alu data sets.

The most likely number of groups, $k$, was estimated as three and four, respectively, for Alu8 and Alu21 (Table 7). All alternatives could be rejected with a high level of confidence. Individuals were then associated with posterior probabilities to belong to each of the previously identified groups, and were assigned to the most likely group (Table 8; had we chosen to attribute an individual to a group only when one of those posterior probabilities is higher than 50\%, 287 genotypes of the Alu 8 data set and 162 of the Alu22 database would have been unclassified). The results inferred from the two data sets differ markedly. Not only is the number of groups different, but also the geographical ranges of the groups do not overlap. For the Alu8 data set, the analysis suggests the existence of a largely Eurasian group, plus two groups whose distribution is essentially worldwide. Conversely, for the Alu21 data set, all African and most Oceanian genotypes fall into the first group, whereas the other three groups roughly correspond to Asia and the Americas (2), and Eurasia (3 and 4). Using a set of X-chromosome data and the same method, Wilson et al. (2001) identified yet another set of groups (four, roughly corresponding to Europe, New Guinea, Africa, and Asia).

\section{Conclusions}

Previous studies have shown that differences among continental groups represent a rather small fraction of the global STR and RFLP diversity of our species (Barbujani et al. 1997;

Table 7. Estimates of the Number of Groups, $K$, in the Alu Data Sets

\begin{tabular}{lccccc}
\hline & \multicolumn{2}{c}{ Alu8 } & & \multicolumn{2}{c}{ Alu21 } \\
\cline { 2 - 3 } \cline { 5 - 6 } $\boldsymbol{n n n n y y}$ & in $\mathbf{p r}(\mathbf{x} \mid \boldsymbol{k})$ & $\mathbf{p}(\boldsymbol{k} \mid \mathbf{x})$ & & in $\mathbf{p r}(\mathbf{x} \mid \boldsymbol{k})$ & $\mathbf{p}(\boldsymbol{k} \mid \mathbf{x})$ \\
\hline 1 & -12560 & $\sim 0$ & & -9700 & $\sim 0$ \\
2 & -12072 & $\sim 0$ & & -9700 & -0 \\
3 & -11974 & -1 & & -9585 & 0.05 \\
4 & -12048 & -0 & & -9582 & 0.95 \\
5 & -12050 & $\sim 0$ & & -9668 & $\sim 0$ \\
\hline
\end{tabular}

Under the assumption of Hardy-Weinberg equilibrium, $\operatorname{In} \operatorname{Pr}(\mathrm{X} I K)$ is the likelihood of the data, given $K$, and $\mathrm{P}(K \mid \mathrm{X})$ is the posterior probability of $K$, given the data.
Jorde et al. 2000; Brown and Armelagos 2001). In this study we found that a between-continent variance accounting for $5 \%$ to $20 \%$ of the total is the rule also for numerous nuclear biallelic polymorphisms, on the basis of independent loci typed in a large number of samples. We identified an exception, Y-chromosome polymorphisms, and we tried to better understand the evolutionary meaning of both the rule and the exception. For that purpose, we asked what is the probability of allocating an individual to the correct continent, on the basis of her or his genotype. Different statistical methods gave somewhat different results, but three conclusions appear justified: (1) most individuals are allocated correctly, but (2) the rate of misclassification is never $<27 \%$, and (3) the rate of misclassification is roughly the same, whether allocation is based on autosomal or Y-chromosome polymorphisms, although for the latter the variance among continents is four times as large. New Y-chromosome data sets containing many new polymorphisms are being assembled (Underhill et al. 2000; Hammer et al. 2001), and their analysis may somewhat modify details of this picture.

Continent-specific and population-specific polymorphisms do exist in humans, and individuals carrying certain, generally pathologic, alleles, can be assigned to a specific geographic area with a high degree of confidence. Popular examples are the alleles for Tay-Sachs disease among Ashkenazi Jews, and for thalassemia in the Mediterranean area. However (with one exception, the Duffy-null alleles in Africa), very few members of those populations carry those rare alleles, and the mutations that generated them are recent (Oddoux et al. 1999; Hamblin and Di Rienzo 2000; Weatherall 2001). Alleles common in a continent, and absent or nearly so elsewhere, which would support the existence of a substantial ancestral differentiation among human groups, have been identified in this or previous studies only in the Y chromosome. Even the $\mathrm{X}$-chromosome haplotypes that initially appeared to be restricted, respectively, to African and to non-African populations (Harris and Hey 1999), turned out to be shared across continents when sample sizes were increased ( $\mathrm{Yu}$ and $\mathrm{Li}$ 2000).

In summary, discriminant analysis confirms the existence of some degree of geographical structuring in humans, contra Templeton (1999). If one considers a set of biallelic loci from an individual's genome, and asks which continent that genotype comes from, the answer will be correct most of the time. However, even when jointly considered, all of the markers we could use, including those of the Y chromosome, did not prove able to assign more than $70 \%$ of the individuals to their continent of origin. That is not what one would expect, 
Table 8. Assignment of Individuals to the Groups Identified by STRUCTURE

\begin{tabular}{|c|c|c|c|c|c|c|c|}
\hline & \multicolumn{3}{|c|}{ Alu8 } & \multicolumn{4}{|c|}{ Alu21 } \\
\hline & group 1 & group 2 & group 3 & group 1 & group 2 & group 3 & group 4 \\
\hline Americas & 9 & 85 & 6 & 0 & 84 & 8 & 8 \\
\hline Oceania & 13 & 50 & 35 & 78 & 9 & 4 & 9 \\
\hline Europe & 51 & 19 & 30 & 6 & 22 & 36 & 36 \\
\hline Asia & 31 & 38 & 31 & 13 & 46 & 18 & 23 \\
\hline Africa & 12 & 50 & 38 & 100 & 0 & 0 & 0 \\
\hline
\end{tabular}

if the human species were subdivided, and deep genetic discontinuities existed among continental groups. We have also shown, albeit in a relatively small sample, that the genetic variances among continents at a locus undergoing selection, $\beta$-globin, are not greater than those estimated at neutral loci.

The genetic uniformity of the human species contrasts with what is observed for other large mammals (reviewed in Templeton 1999), whose populations tend to be more diverse, even when restricted to a much narrower geographic range. Groups occupying distinct territories, and each characterized by peculiar combinations of genes that are absent or at least rare elsewhere, can be found among gorilla (Ruvolo et al. 1994), chimpanzee and bonobo (Gagneaux et al. 1999), gray wolf and elephant (Templeton 1999), and gazelle (Arctander et al. 1996), but not, so far, in humans. Two, not necessarily alternative, explanations seem reasonable, namely: (1) a comparatively recent common ancestry of all modern humans, so that there has been little time for groups to diverge, and (2) gene flow rates high enough to homogenize groups.

Our attempt to identify major human groups by clustering genotypes yielded contradictory results. Different numbers of groups, and different distributions of genotypes within such groups, were observed. Moreover, these results do not overlap with those of another study (Wilson et al. 2001) based on the same method and different data. These observations mean that there is no reason to expect that the same groups will be identified on the basis of different sets of genes. As a consequence, both for evolutionary studies and practical applications (such as predicting liability to certain diseases or response to certain drugs), what seems to matter is the individual genotype, much more than the ethnic or geographic affiliation.

This study shows that, by assuming homogeneity of individuals within their continent, one disregards between $8 \%$ (as estimated in the Alu21 data set) and17\% (Y99 data set) of the total biallelic human diversity (Table 2). The practical consequences of that depend on the composition of the population studied, and may be trivial in forensic applications, especially if STR markers are used, if the populations are homogeneous. However, the error may not be negligible in metropolitan areas, or where very different communities coexist. In those cases, up to $30 \%$ of individuals (Table 3) may carry genotypes that appear so different from the bulk of the others that discriminant analysis would assign them to another continent. As for clinical practice, it seems a clear distinction should be made between population-specific polymorphisms (which exist, albeit rare, and may be a useful diagnostic tool), and continent- or (the term could then be appropriate) race-specific genetic polymorphisms. To the best of our knowledge, no example of the latter has been described in humans. Going back to the evolutionary definition of race that we cited in the Introduction, this study found no evidence suggesting the existence in humans of recognizable lineages that have diverged because they have long been separated by reproductive barriers. The present study of biallelic, presumably ancient, polymorphisms does not suggest that there is a basis for an objective and unequivocal definition of distinct biological groups within the human species.

\section{METHODS}

\section{DNA Samples and Alu Genotyping}

Twenty-one Alu insertion polymorphisms were typed in 1330 individuals from 32 populations (listed in Table 7 along with the sample sizes). The cell lines used to isolate control DNA samples were as follows: human (Homo sapiens), HeLa (ATCC CCL2); chimpanzee (Pan troglodytes), Wes (ATCC CRL1609); gorilla (Gorilla gorilla), Ggo-1 (primary gorilla fibroblasts) provided by Dr. Stephen J. O'Brien, National Cancer Institute, Frederick, MD, USA. Cell lines were maintained as directed by the source and DNA isolations were performed using Wizard genomic DNA purification (Promega). Diverse human DNA samples were isolated from peripheral blood lymphocytes (Ausubel et al. 1996), most of which had been collected for previous studies (Stoneking et al. 1997; Nasidze and Stoneking 2001). African-American, Bantu speakers, HispanicAmerican, Hungarian, Syrian, and Yanomamo DNA samples were available in Batzer's laboratory.

PCR amplification of each Alu insertion polymorphism was performed in 25- $\mu \mathrm{L}$ reactions using 50-100 ng of target DNA, $40 \mathrm{pM}$ of each oligonucleotide primer, $200 \mu \mathrm{M}$ dNTPs in $50 \mathrm{mM} \mathrm{KCl}, 1.5 \mathrm{mM} \mathrm{MgCl} 2,10 \mathrm{mM}$ Tris- $\mathrm{HCl}(\mathrm{pH} 8.4)$ and Taq DNA polymerase (1.25 units), as recommended by the supplier (Life Technologies). Each sample was subjected to the following amplification cycle: an initial denaturation of 2:30

Table 9. Sample Sizes (Individuals) by Continental Group for Each Data Set

\begin{tabular}{lrrrrr}
\hline & \multicolumn{5}{c}{ Data set } \\
\cline { 2 - 6 } Group & Y98 & Y99 & Alu8 & Alu21 & BGL \\
\hline Africa & 380 & 348 & 170 & 130 & 103 \\
Asia & 787 & 1168 & 883 & 266 & 67 \\
Europe & 217 & 175 & 657 & 650 & 46 \\
Americas & 44 & 389 & 198 & 164 & 48 \\
Australia & 116 & 118 & 162 & 120 & 85 \\
\hline Total & 1544 & 2198 & 2070 & 1330 & 349 \\
\hline
\end{tabular}




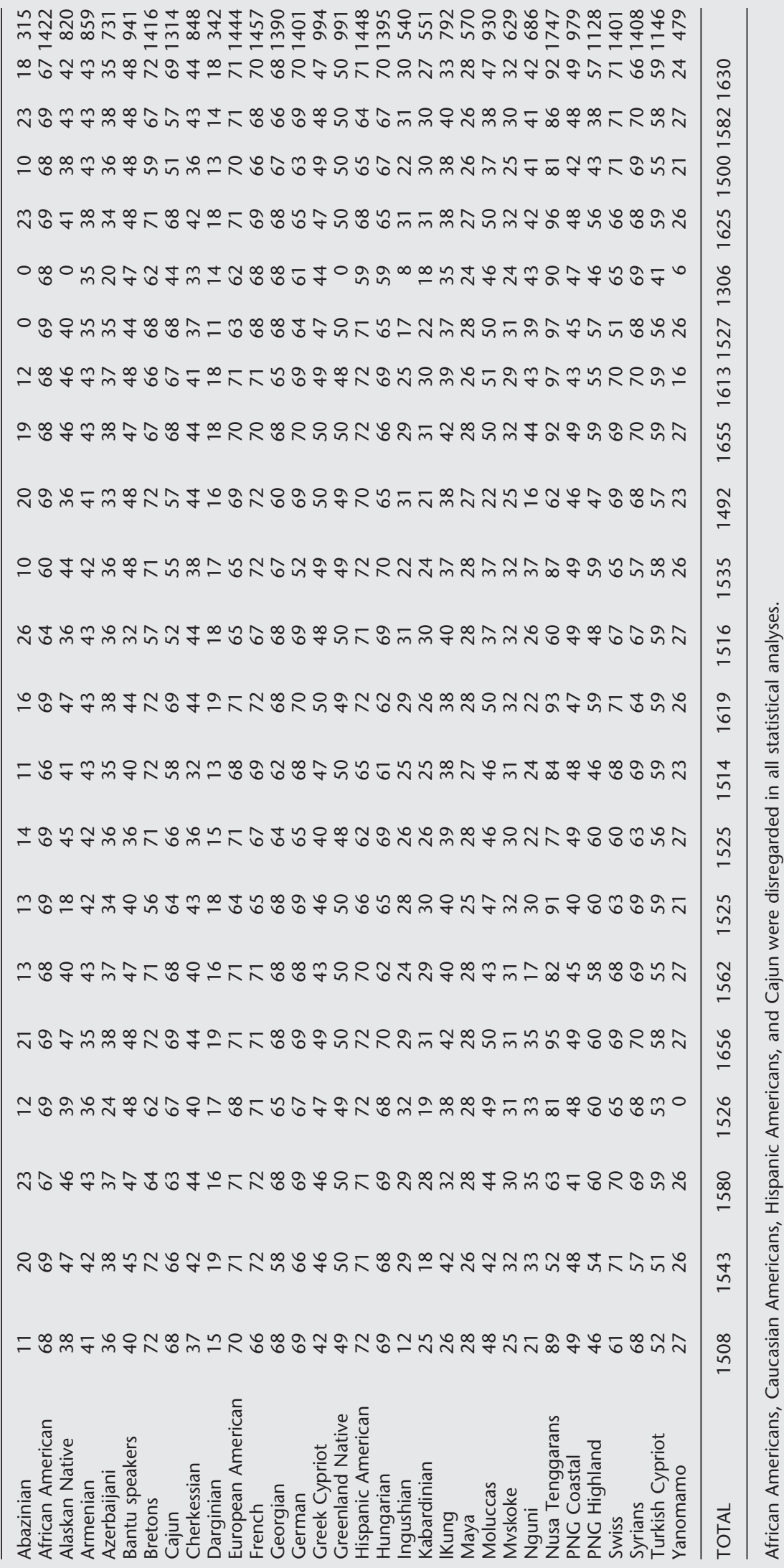




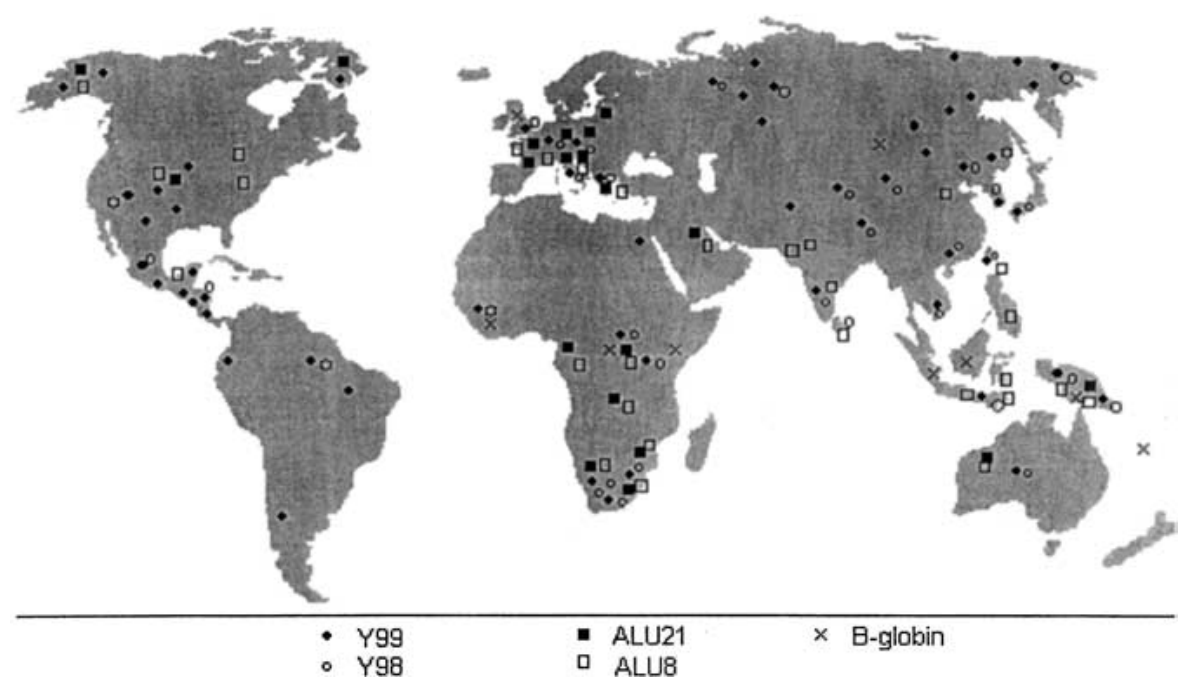

Figure 2 Geographical distribution of the five data sets.

$\min$ at $94^{\circ} \mathrm{C}, 1 \mathrm{~min}$ of denaturation at $94^{\circ} \mathrm{C}, 1 \mathrm{~min}$ at the annealing temperature, $1 \mathrm{~min}$ of extension at $72^{\circ} \mathrm{C}$, repeated for 32 cycles, followed by a final extension at $72^{\circ} \mathrm{C}$ for $10 \mathrm{~min}$. Twenty microliters of each sample was fractionated on a $2 \%$ agarose gel with $0.25 \mu \mathrm{g} / \mathrm{mL}$ ethidium bromide. PCR products were directly visualized using UV fluorescence. The sequences of the oligonucleotide primers, annealing temperatures, PCR product sizes, and chromosomal locations for the loci have been reported previously (Arcot et al. 1996, 1997, 1998; Stoneking et al. 1997).

\section{Data Sets}

Five data sets were considered in this study (Table 9). The Alu21 data set represents the newly typed, unlinked autosomal Alu insertion polymorphisms in the 32 populations of Table 10.

Four additional data sets were assembled and analyzed. The Alu8 data set includes Alu insertion genotypes at 8 autosomal loci (TPA25, PV92, APO, ACE, FXIIIB, D1, A25, B65), with 1500 individuals from 32 worldwide populations (Stoneking et al. 1997), representing 1331 distinct multilocus genotypes. Further populations were incorporated into the Alu8 database, bringing the total number of populations to 46. Although all loci of the Alu8 data set are also found in the Alu21 data set, the populations are not the same (for example Eastern Asian populations are absent from Alu21), and therefore we chose to analyze the two databases separately. The Y98 and Y99 data sets are drawn from two surveys (Hammer et al. 1998; Karafet et al. 1999) of 12 biallelic Y-chromosome polymorphisms, defining respectively 12 and 14 distinct haplotypes in 1544 males from 35 populations (Y98), and 2198 males from 60 populations (Y99). The final data set comes from a study of a 3 -kb region encompassing the $\beta$-globin gene in nine populations (Harding et al. 1997) (BGL data set). The gene tree constructed from 326 sequences includes 29 haplotypes, 13 of them apparently resulting from recombination or gene conversion. The latter haplotypes are all rare, and to avoid choosing arbitrary weights for nucleotide substitution, recombination, and gene conversion events, we chose to consider only the remaining 16 haplotypes, assuming they have been generated by nucleotide substitution alone.

The geographical distribution of the samples for each data set is shown in Figure 2. The Y99 and Y98 data sets have the widest global coverage. Alu8 has samples almost everywhere apart from North Asia, whereas Alu21 and BGL are smaller data sets; in particular, for most continents, BGL has only one sample.

\section{Statistical Analysis: AMOVA}

The genetic differences within and among population samples were quantified, and their significance was assessed, using AMOVA, a nonparametric method for the analysis of variance suitable for molecular data (Excoffier et al. 1992). Genetic variances were estimated from allele-frequency differences between populations, and from measures of molecular difference between alleles. The overall genetic variance was then subdivided into three hierarchical components: between individuals within populations, between populations of the same group, and between groups. Because morphological studies of the last two centuries led to lists of human races containing from 3 to 200 items (Armelagos 1994; Barbujani 2001), and in the absence of other solid criteria for group definition, we decided to use groups corresponding to continents. The significance of the variance components was tested by a randomization approach. Each individual, or population, was reassigned to a random location, according to three resampling schemes. The molecular variances were recalculated, and the procedure was repeated 1000 times to obtain empirical null distributions of all relevant variances.

\section{Statistical Analysis: Discriminant Analysis}

In discriminant analysis, also known as supervised classification (Ripley 1996), variables measured on individuals whose grouping is known (the training data set) are combined to construct a new variable that can be used to classify individuals (or, in our case, genotypes) of unknown group (query genotypes). For the analyses described following, we have used the classification functions implemented for the statistical package Splus that are freely available on the StatLib server ( $S$ Archive: http://lib.stat.cmu.edu/) (Venables and Ripley 1997). The genotypes were coded as strings of binary digits, so that distances estimated between pairs of individuals reflected the minimum (and most likely) number of mutational events separating them. This coding allows one to use both parametric and nonparametric forms of discriminant analysis.

We initially considered three standard parametric methods, namely, linear (LDA), logistic (LOG), and quadratic (QDA) discriminant analysis. All of these assume that the variables are at least approximately normal, which does not hold for our data. They performed poorly and are not discussed further here, although some results are included following for comparison. We then resorted to four standard nonparametric methods, which do not assume a probabilistic model for the observations, namely, a neural network (NNET), Gaussian kernel density estimation (KER), and $k$-nearest neighbor with $k=1(1 \mathrm{NN})$ and $k=3$ (3NN) (Venables and Ripley 1997).

Neural networks are collections of mathematical models, and related computer programs, which identify patterns in a data set by emulating some properties of biological nervous systems and by drawing on the analogies of adaptive biological learning (Jennions and Brooks 2001). NNETs are composed of a large number of interconnected processing elements that are analogous to neurons, and are tied together with weighted connections that are analogous to synapses. By a process of trial and error, nonlinear functions are estimated 
from randomly sampled subsets of the original data set. These functions are then used by NNETs to classify the remaining genotypes. The goodness of the classification obtained is evaluated, and iterations are run until a desired level of accuracy is obtained.

The KER method starts by estimating the density of the genotype frequencies in each group, and then assigns a new observation (genotype) to the group for which its estimated density is maximal. Lastly, with the simplest method, the nearest neighbor, each genotype is assigned to the group whose first $k$ nearest genotypes (one for what we refer to as $1 \mathrm{NN}$, or three for $3 \mathrm{NN}$ ) are closest.

The previously described classification methods are not specifically designed for genetic data. We also implemented a method (RM) that, for the autosomal data sets, exploits the assumptions of Hardy-Weinberg and linkage equilibria (independence within and between loci) to improve the estimation of genotype relative frequencies in each group (Rannala and Mountain 1997). The RM method uses Bayesian posterior expectations given a symmetric Dirichlet prior distribution to overcome the potential problem of zero frequency in the training data for an allele observed in the query genotype. In the case of nonrecombining haploid data, the equilibrium assumptions are not appropriate, and the natural analog of the RM method reduces to a trivial comparison of (posterior) haplotype frequencies.

For the Alu8 and Alu21 data sets, we included only those individuals with complete information (over all 8 or 21 loci), reducing the sample sizes to 1331 and 477 individuals, respectively. For these data sets, the variables involved in the discriminant analysis are the individual genotypes at each locus. For the other data sets (BGL, Y98 and Y99), the entire haplotype is treated as a single variable. One by one, each individual's known source population is temporarily ignored, and each of the classification methods is implemented to classify that individual into her or his most likely source population, with all the other individuals being used as the training set. At the end of this cross-validation procedure, the proportion of correct continental allocations was recorded.

\section{Statistical Analysis: Inference of Population Structure}

We estimated the most likely number of genetically homogeneous groups in the data sets, and assigned each individual to her or his most likely group by means of an approach implemented in the program STRUCTURE (Pritchard et al. 2000). Multilocus genotypes are considered, and no particular mutational model is assumed. Each individual's genotype is considered to result from a mixture of contributions originating in $k$ population groups, and $q(i)$ is the fraction of the genes of that individual that come from the $i$ th group defined. Under the assumption that each of the populations is in HardyWeinberg equilibrium, $k$ is estimated by a Monte CarloMarkov Chain algorithm. Then, for each individual, regardless of her or his geographical provenance, the vector $q(1)$, $q(2) \ldots q(k)$ is estimated, and ultimately each individual can be assigned to one of the inferred groups, that is, the one with the highest probability.

\section{ACKNOWLEDGMENTS}

We thank Walter Fitch, Giorgio Bertorelle, Ryan Brown, George Armelagos, Joseph Terwilliger, Lorena Madrigal, and two anonymous reviewers for many comments and suggestions. This research was supported by grants from the University of Ferrara, from the Lousiana Board of Regents Millennium Trust Health Excellence Fund HEF (2000-05)-05, (2000-05)-01, and (2001-06)-02 (MAB), and awards 1999-IJCX-K009 and 2001-IJ-CX-K004 from the Office of Justice Programs, National Institute of Justice, Department of Justice (MAB). A 9-month's stay of C.R. at the University of Reading was partly supported by funds of the Department of Applied
Statistics. Points of view in this document are those of the authors and do not necessarily represent the official position of the U.S. Department of Justice.

The publication costs of this article were defrayed in part by payment of page charges. This article must therefore be hereby marked "advertisement" in accordance with 18 USC section 1734 solely to indicate this fact.

\section{REFERENCES}

Arcot, S.S., Adamson, A.W., Lamerdin, J.E., Kanagy, B., Deininger P.L., Carrano, A.V., and Batzer, M.A. 1996. Alu fossil relics-Distribution and insertion polymorphism. Genome Res. 6: $1084-1092$.

Arcot, S.S., DeAngelis, M.M., Sherry, S.T., Adamson, A.W., Lamerdin, J.E., Deininger, P.L., Carrano, A.V., and Batzer, M.A. 1997. Identification and characterization of two polymorphic Ya5 Alu repeats. Mutat. Res. Genomics 382: 5-11.

Arcot, S.S., Adamson, A.W., Risch, G., LaFleur, J., Lamerdin, J.E., Carrano, A.V., and Batzer, M.A. 1998. High-resolution cartography of recently integrated chromosome 19-specific Alu fossils. J. Mol. Biol. 281: 843-855.

Arctander, P., Kat, P.W., Aman, R.A., and Siegismund, H.R. 1996. Extreme genetic differences among populations of Gazella granti, Grant's gazelle, in Kenya. Heredity 76: 465-475.

Armelagos, G.J. 1994. Racism and physical anthropology: Brues's review of Barkan's The retreat of scientific racism. Am. J. Phys. Anthropol. 93: 381-383.

Ausubel, L.J., Kwan, C.K., Sette, A., Kuchroo, V., and Hafler, D.A. 1996. Complementary mutations in an antigenic peptide allow for crossreactivity of autoreactive T-cell clones. Proc. Natl. Acad. Sci. 93: 15317-15322.

Barbujani, G. 2001. What genetics tells us about races. In International encyclopedia of the social and behavioral sciences (eds. N.J. Smelser and P.B. Baltes), Vol. 19, pp. 12694-12700. Elsevier Science, Oxford.

Barbujani, G., Magagni, A., Minch, E., and Cavalli-Sforza, L.L. 1997. An apportionment of human DNA diversity. Proc. Natl. Acad. Sci. 94: $4516-4519$

Benar, V.B., Lee, N.C., Piper, M., and Richardson, L. 2001. Race-specific results of Papanicolaou testing and the rate of cervical neoplasia in the National Breast and Cervical Cancer Early Detection Program, 1991-1998 (United States). Cancer Causes Control 12: 61-68.

Brown, R.A. and Armelagos, G.J. 2001. Apportionment of racial diversity: A review. Evol. Anthropol. 10: 34-40.

Cavalli-Sforza, L.L., Menozzi, P., and Piazza, A. 1994. The history and geography of human genes. Princeton University Press, Princeton.

Deka, R., Guangyun, S., Smelser, D., Zhong, Y., Kimmel, M., and Chakraborty, R. 1999. Rate and directionality of mutations and effects of allele size constraints at anonymous, gene-associated, and disease-causing trinucleotide loci. Mol. Biol. Evol. 16: $1166-1177$.

Estrada, D.A. and Billett, H.H. 2001. Racial variation in fasting and random homocysteine levels. Am. J. Hematol. 66: 252-256.

Excoffier, L., Smouse, P.E., and Quattro, J.M. 1992. Analysis of molecular variance inferred from metric distances among DNA haplotypes: Application to human mitochondrial DNA restriction data. Genetics 131: 479-491.

Gagneux, P., Wills, C., Gerloff, U., Tautz, D., Morin, P.A., Boesch, C. Fruth, B., Hohmann, G., Ryder, O.A., and Woodruff, D.S. 1999. Mitochondrial sequences show diverse evolutionary histories of African hominoids. Proc. Natl. Acad. Sci. 96: 5077-5082.

Gill, P. and Evett, I. 1995. Population genetics of short tandem repeat (STR) loci. Genetica 96: 69-87.

Hamblin, M.T. and Di Rienzo, A. 2000. Detection of the signature of natural selection in humans: Evidence from the Duffy blood group locus. Am. J. Hum. Genet. 66: 1669-1679.

Hammer, M.F., Karafet, T., Rasanayagam, A., Wood, E.T., Altheide, T.K., Jenkins, T., Griffiths, R.C., Templeton, A.R., and Zegura S.L. 1998. Out of Africa and back again: Nested cladistic analysis of human Y chromosome variation. Mol. Biol. Evol. 15: 427-441.

Hammer, M.F., Karafet, T., Redd, A.J., Jarjanazi, H. Santachiara-Benerecetti, S., Soodyall, H., and Zegura, S.L. 2001. Hierarchical patterns of global human Y-chromosome diversity. Mol. Biol. Evol. 18: 1189-1203.

Harding, R.M., Fullerton, S.M., Griffiths, R.C., Bond, J., Cox, M.J., Schneider, J.A., Moulin, D.S., and Clegg, J.B. 1997. Archaic African and Asian lineages in the genetic ancestry of modern 
humans. Am. J. Hum. Genet. 60: 772-789.

Harris, E.E. and Hey, J. 1999. X chromosome evidence for ancient human histories. Proc. Natl. Acad. Sci. 96: 3320-3324.

Hartz, R.S., Rao, A.V., Plomondon, M.E., Grover, F.L., and Shroyer, A.L. 2001. Effects of race, with or without gender, on operative mortality after coronary artery bypass grafting: A study using The Society of Thoracic Surgeons National Database. Ann. Thorac. Surg. 71: 512-520.

Hoffman, R.M., Gilliland, F.D., Eley, J.W., Harlan, L.C., Stephenson, R.A., Stanford, J.L., Albertson, P.C., Hamilton, A.S., Hunt, W.C., and Potosky, A.L. 2001. Racial and ethnic differences in advanced-stage prostate cancer: The prostate cancer outcomes study. J. Natl. Cancer Inst. 93: 388-395.

Jennions, M.D. and Brooks, R. 2001. A sense of history. Trends Ecol. Evol. 16: 113-115.

Jobling, M.A., Pandya, A., and Tyler-Smith, C. 1997. The Y chromosome in forensic analysis and paternity testing. Int. J. Legal Med. 110: 118-124.

Jorde, L.B., Watkins, W.S., Bamshad, M.J., Dixon, M.E., Ricker, C.E., Seielstad, M.T., and Batzer, M.A. 2000. The distribution of human genetic diversity: A comparison of mitochondrial, autosomal and Y-chromosome data. Am. J. Hum. Genet. 66: 979-988.

Karafet, T.M., Zegura, S.L., Posukh, O., Osipova, L., Bergen, A., Long J., Goldman, D., Klitz, W., Harihara, S., de Knijff, P., et al. 1999. Ancestral Asian source(s) of new world Y-chromosome founder haplotypes. Am. J. Hum. Genet. 64: 817-831.

Kayser, M. and Sajantila, A. 2001. Mutations at Y-STR loci: Implications for paternity testing and forensic analysis. Forensic Sci. Int. 118: 116-121.

Lander, E.S. and Budowle, B. 1994. DNA fingerprinting dispute laid to rest. Nature 371: 735-738.

Latter, B.D.H. 1980. Genetic differences within and between populations of the major human subgroups. Am. Nat. 116: $220-237$.

Lewontin, R.C. 1972. The apportionment of human diversity. Evol. Biol. 6: 381-398.

Markovtsova, L., Marjoram, P., and Tavare, S. 2000. The age of a unique event polymorphism. Genetics 156: 401-409.

Morton, N.E. 1994. Genetic structure of forensic populations. Am. J. Hum. Genet. 55: 587-588.

Nasidze, I. and Stoneking, M. 2001. Mitochondrial DNA variation and language replacements in the Caucasus. Proc. R. Soc. Lond. B Biol. Sci. 268: 1197-1206.

National Research Council. 1992. DNA technology in forensic science. National Academy Press, Washington, DC.

Oddoux, C., Guillen-Navarro, E., Ditivoli, C., Dicave, E., Cilio, M.R., Clayton, C.M., Nelson, H., Sarafoglou, K., McCain, M., Peretz, H., et al. 1999. Mendelian diseases among Roman Jews: Implications for the origins of disease alleles. J. Clin. Endocrinol. Metab. 84: $4405-4409$.

Pennock, D.S. and Dimmick, W.W. 1997. Critique of the evolutionarily significant unit as a definition for distinct population segments under the US Endangered Species Act. Conserv. Biol. 11: 611-619.

Pritchard, J.K., Stephens, M., and Donnelly, P. 2000. Inference of population structure using multilocus genotype data. Genetics
155: 945-959.

Rannala, B. and Mountain, J.L. 1997. Detecting immigration by using multilocus genotypes. Proc. Natl. Acad. Sci.94: 9197-9201.

Relethford, J. 1994. Craniometric variation among modern human populations. Am. J. Phys. Anthropol. 95: 53-62.

Ripley, B.D. 1996. Pattern recognition and neural networks. Cambridge University Press, Cambridge.

Roeder, K. 1994. DNA fingerprinting: A review of the controversy. Stat. Science 9: 222-278.

Ruvolo, M., Pan, D., Zehr, S., Goldberg, T., Disotell, T.R., and von Dornum, M. 1994. Gene trees and hominoid phylogeny. Proc. Natl. Acad. Sci. 91: 8900-8904.

Seielstad, M., Minch, E., and Cavalli-Sforza, L.L. 1998. Genetic evidence for a higher female migration rate in humans. Nat. Genet. 20: $278-280$.

Shaw, B.A. and Krause, N. 2001. Exploring race variations in aging and personal control. J. Gerontol. B Psychol. Sci. Soc. Sci. 56: S119-124.

Stoneking, M., Fontius, J.J., Clifford, S.L., Soodyall, H., Arcot, S.S., Saha, N., Jenkins, T., Tahir, M.A., Deininger, P.L., and Batzer, M.A. 1997. Alu insertion polymorphisms and human evolution Evidence for a larger population size in Africa. Genome Res. 7: 1061-1071.

Templeton, A.R. 1999. Human races: A genetic and evolutionary perspective. Am. Anthropol. 100: 632-650.

Underhill, P.A., Shen, P., Lin, A.A., Passarino, G., Yang, W.H., Kauffman, E., Bonné-Tamir, B., Bertranpetit, J., Francalacci, P., Ibrahim, M., et al. 2000. Y chromosome sequence variation and the history of human populations. Nat. Genet. 26: 358-361.

Venables, W.N. and Ripley, B.D. 1997. Modern applied statistics with S-PLUS, 2d ed. Springer Verlag, Berlin.

Watkins, W.S., Ricker, C.E., Bamshad, M.J., Carroll, M.L., Nguyen, S.V., Batzer, M.A., Harpending, R.C., Rogers, A.R., and Jorde, L.B. 2001. Patterns of ancestral human diversity: An analysis of Alu-insertion and restriction-site polymorphisms. Am. J. Hum. Genet. 68: 738-752.

Weatherall, D.J. 2001. Phenotype-genotype relationships in monogenic disease: Lessons from the thalassaemias. Nat. Rev. Genet. 2: 245-255

Weir, B.B. 2001. Forensics. In Handbook of statistical genetics (eds. D.J. Balding, M. Bishop, and C. Cannings), pp. 721-739. John Wiley \& Sons, Chichester, UK.

Wilson, J.E., Weale, M.E., Smith, A.C., Gratrix, F., Fletcher, B., Thomas, M.G., Bradman, N., and Goldstein, D.B. 2001. Population genetic structure of variable drug response. Nat. Genet. 29: 265-269.

Wright, S. 1969. In Evolution and the genetics of populations., Vol. 2, The theory of gene frequencies. Chicago University Press, Chicago.

Yu, N. and Li, W.S. 2000. No fixed nucleotide difference between Africans and non-Africans at the pyruvate dehydrogenase E1 $\alpha$-subunit locus. Genetics 155: 1481-1483.

Received September 18, 2001; accepted in revised form February 12, 2002.

\section{Genome Research}




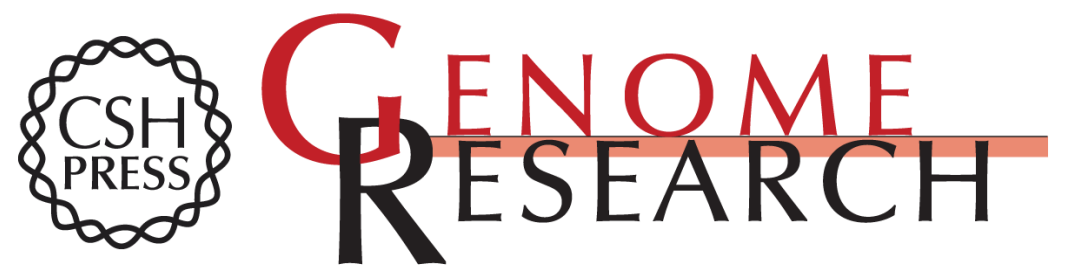

\section{Patterns of Human Diversity, within and among Continents, Inferred from Biallelic DNA Polymorphisms}

Chiara Romualdi, David Balding, Ivane S. Nasidze, et al.

Genome Res. 2002 12: 602-612

Access the most recent version at doi:10.1101/gr.214902

References This article cites 45 articles, 12 of which can be accessed free at:

http://genome.cshlp.org/content/12/4/602.full.html\#ref-list-1

\section{License}

Email Alerting Receive free email alerts when new articles cite this article - sign up in the box at the Service top right corner of the article or click here.

\section{Affordable, Accurate Sequencing.}

